\title{
Effects of mild induced hypothermia on hippocampal connexin 43 and glutamate transporter 1 expression following traumatic brain injury in rats
}

\author{
YUE-HONG LI ${ }^{1}$, CHUN-LAI ZHANG ${ }^{2}$, XIAO-YAN ZHANG ${ }^{3}$, HONG-XIA ZHOU ${ }^{3}$ and LING-LI MENG ${ }^{3}$ \\ Departments of ${ }^{1}$ Obstetrics and Gynecology, and ${ }^{2}$ Cardiology, Tangshan Gongren Hospital, Tangshan, Hebei 063000; \\ ${ }^{3}$ School of Basic Medical Sciences, Hebei United University, Tangshan, Hebei 063000, P.R. China
}

Received March 16, 2014; Accepted June 17, 2014

DOI: $10.3892 / \mathrm{mmr} .2014 .2928$

\begin{abstract}
Traumatic brain injury (TBI) is a common cause of worldwide disability and mortality. Currently, the incidence and prevalence of TBI is markedly increasing and an effective therapy is lacking. Therapeutic hypothermia $\left(32-35^{\circ} \mathrm{C}\right)$ has been reported to reduce intracranial pressure and induce putative neuroprotective effects. However, the underlying molecular mechanisms remain to be elucidated. The aim of the present study was to investigate the effects of mild induced hypothermia (MIH) on the expression of connexin 43 (Cx43) and glutamate transporter 1 (GLT-1) in the hippocampus following TBI in rats. A rat model of TBI was created using a modified weight-drop device, followed by $4 \mathrm{~h}$ of hypothermia $\left(33^{\circ} \mathrm{C}\right)$ or normothermia $\left(37^{\circ} \mathrm{C}\right)$. A wet-dry weight method was used to assess brain edema and spatial learning ability was evaluated using a Morris water maze. The levels of Cx43 and GLT-1 were detected by immunohistochemical and western blot analysis, respectively. The results demonstrated that $\mathrm{MIH}$ treatment improved TBI-induced brain edema and neurological function deficits. In addition, therapeutic MIH significantly downregulated $\mathrm{Cx} 43$ expression and upregulated the levels of GLT-1 in the hippocampus post-TBI. These findings suggested that treatment with MIH may provide a novel neuroprotective therapeutic strategy for TBI through reversing the increase in Cx43 protein and the decrease in GLT-1.
\end{abstract}

Correspondence to: Dr Chun-Lai Zhang, Department of Cardiology, Tangshan Gongren Hospital, 27 Wenhua Road, Tangshan, Hebei 063000, P.R. China

E-mail: zhangchunlai1970@yeah.net

Abbreviations: TBI, traumatic brain injury; MIH, mild induced hypothermia; Cx43, connexin 43; GLT-1, glutamate transporter 1; $\mathrm{TH}$, therapeutic hypothermia; CNS, central nervous system

Key words: traumatic brain injury, mild induced hypothermia, brain edema, connexin 43 , glutamate transporter 1

\section{Introduction}

Mild induced hypothermia (MIH) is considered to improve survival rate and provide a potential neuroprotective effect in acute neurological injury, with favorable neurological outcomes. Several studies have identified MIH as a potential therapeutic strategy for the treatment of traumatic brain injury (TBI) $(1,2)$. The first study of MIH as a treatment option for patients with TBI was reported in 1945 (3). MIH, as a non-pharmacological measure, exerts a complex range of effects in the pathophysiological process of TBI. More specifically, MIH treatment may not only improve histopathological and behavioral outcomes, but also has the ability to reduce intracranial pressure in animal models and clinical trials of TBI $(4,5)$. However, the molecular and cellular mechanisms underlying hypothermic protection remain to be elucidated. The neuroprotective mechanism of MIH is initially attributed to a reduction in the cerebral metabolic rate of oxygen (6). Several studies over the last decade have demonstrated that MIH was able to significantly enhance glucose utilization, inhibit free radical production and suppress inflammatory responses and apoptotic pathways. Additionally, previous studies have indicated that therapeutic MIH may improve traumatic outcomes, including the modulation of temperature-sensitive microRNA expression and suggested that MIH has a beneficial effect on the gene expression profile of the hippocampus following TBI in rats (7). On the basis of these findings, the present study assessed the hypothesis that MIH treatment has the ability to regulate the expression of connexin $43(\mathrm{Cx} 43)$ and glutamate transporter 1 (GLT-1) in the hippocampus following TBI in rats.

Gap junctions, composed of proteins from the connexin family, enable intercellular communication between cells. It has been reported that gap junctions have important regulatory effects in essential cellular processes, including electrical coupling, metabolic transport, proliferation, differentiation and apoptosis (8). Cx43 is the most widely and highly expressed gap junction protein in several different tissues (9). In the central nervous system, Cx43 is mainly expressed in astrocytes and forms the major component protein in astrocytic gap junctions. In addition, astrocytes establish a glial network and communicate through gap junctions in the brain, indicating a potential 
neuroprotective role of astrocytic gap junctions. Mroue et al found that alterations in the expression of $\mathrm{Cx} 43$ could affect cellular fate (10) and several studies have demonstrated that Cx43 had a physiological and pathophysiological impact on a variety of systems $(11,12)$. In addition, results from our previous study demonstrated that TBI stimulated the increased expression of $\mathrm{Cx} 43$ on the surface of astrocytes in rats (13). Ohsumi et al also investigated the expression and distribution of astrocytic Cx43 gap junctions in the hippocampus and the cortex following TBI, suggesting that $\mathrm{Cx} 43$ participates in the pathophysiological processes of brain damage (14). Notably, other studies have revealed that $\mathrm{Cx} 43$ is significantly enhanced in the selectively vulnerable CA1-CA2 region of the hippocampus following brain injury (15).

In addition to being gap-junctional proteins, connexins may exist in the form of unopposed halves of gap junction channels, termed hemichannels (16). Cx43-hemichannels in astrocytic gap junctions remain closed under normal physiological conditions but they may open following brain injury. Astrocytes have been demonstrated to release relatively large molecules, including adenosine triphosphate and glutamate, by the opening of Cx43-hemichannels, which was involved in the propagation of injury in the brain (17). Glutamate, a major excitatory neurotransmitter, causes excitotoxicity and neuronal cell death at high concentrations. Glutamate transport is the only mechanism involved in extracellular glutamate clearance (18). GLT-1, as the major glutamate transporter, is predominantly expressed in astrocytes. GLT-1 provides the majority of glutamate clearance capacity by transporting it into the intracellular space (19). Previous studies have demonstrated that the mRNA and protein levels of GLT-1 are depressed in the cortex following TBI, which contributes to an increase in extracellular glutamate concentration following TBI (20). Therefore, stimulating an increase in GLT-1 expression following brain injury may improve neuronal damage and functional deficit.

These findings suggest that Cx43 and GLT-1 may be potential targets for the treatment of TBI. In the present study, the potential therapeutic effect of MIH was investigated in a rat model of TBI and the possibility that treatment with $\mathrm{MIH}$ may reduce the increase in $\mathrm{Cx} 43$ expression and reverse post-traumatic GLT-1 suppression in the hippocampus following TBI was assessed.

\section{Materials and methods}

Animals and TBI model. All procedures were performed in accordance with the guidelines of the Chinese Council on Animal Protection and were approved by Hebei United University Committee for the use of animals in research (Tangshan, China). A total of 90 male Sprague-Dawley (SD) rats (aged 12-16 weeks and weighing 350-375 g) were purchased from Vital River Laboratory Animal Technology Co., Ltd. (Bejing, China). The rat model of TBI was induced by using a modified weight-drop device, as described previously by Marmarou et al (21). All animals were anesthetized with pentobarbital sodium (Nembutal; $60 \mathrm{mg} / \mathrm{kg}$ ). A midline incision was made to expose the skull between the bregma and lambda suture lines and a steel disc (diameter, $10 \mathrm{~mm}$; thickness, $3 \mathrm{~mm}$ ) was adhered to the exposed skull using dental acrylic. Animals were moved onto a foam mattress underneath a weight-drop device, in which a $450 \mathrm{~g}$ weight falls freely through a vertical tube from a height of $1.5 \mathrm{~m}$ onto the steel disc. Animals in the sham group underwent the same surgical procedure without weight-drop impact. Following treatment, the rats were individually housed in separate cages. The rats were placed on warming pads for $24 \mathrm{~h}$ post-surgery, to aid them in maintaining a regular body temperature.

Sham, TBI normothermia and MIH treatment groups. The 90 male SD rats were randomly divided into three groups $(n=30)$ : Sham group, TBI normothermia group $\left(37^{\circ} \mathrm{C}\right)$ and $\mathrm{MIH}$-treated group $\left(33^{\circ} \mathrm{C}\right)$. Mild hypothermia was induced, as described previously (22). The control of the temperature was initiated $30 \mathrm{~min}$ post-TBI. Temperature probes were used to measure temporalis muscle and rectal (core) temperature. Selective brain hypothermia was induced 30 min after injury, with a target hypothermic temperature achieved 40 min after TBI and maintained using cooled air and heating lamps for $4 \mathrm{~h}$. Animals were then rewarmed slowly over a $1.5 \mathrm{~h}$ period. Rats were anesthetized and sacrificed at 6, 12, 24, 48 and $72 \mathrm{~h}$ following TBI.

Evaluation of brain edema. Brain edema was evaluated by analysis of brain water content, as described previously (23). Rat brains were separated and weighed immediately with a chemical balance to obtain a wet weight (WW). Following drying in a desiccating oven for $24 \mathrm{~h}$ at $100^{\circ} \mathrm{C}$, dry tissues were weighed again to obtain a constant dry weight (DW). The percentage of water in the tissues was calculated according to the following formula: \% brain water $=(\mathrm{WW}-\mathrm{DW}) / \mathrm{WW}) \times 100$.

Morris water maze test. Spatial learning ability was assessed using a Morris water maze, as described previously (24). The Morris water maze consists of a black circular pool (diameter, $180 \mathrm{~cm}$; depth, $45 \mathrm{~cm}$ ) filled with water to a depth of $30 \mathrm{~cm}$ (temperature, $26^{\circ} \mathrm{C}$ ). The maze is divided into four equivalent quadrants: north $(\mathrm{N})$, west (W), south (S) and east (E). A $2 \mathrm{~cm}$ submerged escape platform $(12 \mathrm{~cm}$ diameter, $28 \mathrm{~cm}$ height, made opaque with paint) was placed in the middle of one of the quadrants, equidistant from the sidewall and the center of the pool. Rats were trained to find the platform prior to TBI or sham surgery. For each trial, the rat was randomly placed at a quadrant start point $(\mathrm{N}, \mathrm{S}, \mathrm{E}$ or $\mathrm{W})$ facing the wall of the pool. The rats were permitted a maximum of $60 \mathrm{sec}$ to escape to a platform, rats which failed to escape within $90 \mathrm{sec}$ were placed on the platform for a maximum of $20 \mathrm{sec}$ and returned to the cage for a new trial (intertrial interval $20 \mathrm{sec}$ ). The escape latency performance was recorded using a video camera and video tracking system (HVS Imaging, Hampton, UK). The average escape latency of a total of five trials was calculated. This test was conducted at 24,48 and $72 \mathrm{~h}$ post TBI.

Immunohistochemical analysis. Immunohistochemical analysis was performed using an SABC immunohistochemistry kit (Wuhan Boster Biological Engineering Co., Ltd., Wuhan, China) according to the manufacturer's instructions. Paraffin embedded brain tissue sections $(5 \mu \mathrm{m})$ were heated for $30 \mathrm{~min}$ at $60^{\circ} \mathrm{C}$, dewaxed and rehydrated, followed by 
microwave antigen retrieval procedures. In brief, sections in Citrate salt buffer (Wuhan Boster Biological Engineering Co., Ltd., Wuhan, China) were heated in a microwave oven for 5 min. Endogenous peroxidase was inactivated with $3 \% \mathrm{H}_{2} \mathrm{O}_{2}$ for $10 \mathrm{~min}$ at room temperature. The sections were incubated in $5 \%$ bovine serum albumin (Gibco BRL, Carlsbad, CA, USA) solution for $20 \mathrm{~min}$, to inhibit the nonspecific binding. Subsequently, sections were incubated overnight at $4{ }^{\circ} \mathrm{C}$ with rabbit anti-Cx43, GLT-1 polyclonal antibodies (Santa Cruz Biotechnology Inc., Santa Cruz, CA, USA) diluted 1:50 and then with polyclonal horseradish peroxidase-conjugated anti-rabbit immunoglobulin G (IgG; Wuhan Boster Biological Engineering Co., Ltd., Wuhan, China) antibodies for $30 \mathrm{~min}$. Diaminobenzidine (Wuhan Boster Biological Engineering Co., Ltd.) was used to reveal the immunohistochemical reaction.

Western blot analysis. Total proteins from the hippocampal CA1 regions were rapidly isolated and extracted. The concentration of total protein was measured using a bicinchoninic acid reagent (Solarbio Science and Technology Co., Ltd., Beijing, China). Samples were subjected to sodium dodecyl sulfate polyacrylamide gel electrophoresis. The proteins separated on the gel were transferred onto polyvinylidene difluoride membranes (Roche Diagnostics $\mathrm{GmBH}$, Mannheim, Germany). Blots were inhibited with 5\% fat-free dry milk for $2 \mathrm{~h}$ at room temperature and were incubated overnight at $4^{\circ} \mathrm{C}$ with rabbit anti-Cx43, GLT- 1 and $\beta$-actin polyclonal antibody (Santa Cruz Biotechnology, Inc.; diluted 1:200). The blots were then incubated with horseradish peroxidase conjugated anti-rabbit IgG (Cell Signaling Technology, Inc., Danvers, MA, USA; diluted 1:5,000) for $2 \mathrm{~h}$ at room temperature. Following incubation, the immunoblot on the membrane was visible following development with an enhanced chemiluminescence detection system (ChemiDoc XRS; Bio Rad, Hercules, CA, USA) and the densitometric signals were quantified using an imaging program (Image Lab 4.1; Bio-Rad). The immunoreactive bands of all proteins expressed were normalized to the intensity of corresponding bands for $\beta$-actin. The results of the western blot analysis were analyzed using National Institutes of Health Image 1.41 software (Bethesda, MD, USA).

Statistical analysis. Data are presented as the mean \pm standard deviation. SPSS 16.0 (SPSS, Inc., Chicago, IL, USA) was used for statistical analysis of the data. Statistical analysis was performed using analysis of variance and subsequently by the Student-Newman-Keuls post-hoc test or Student's t-test (comparison of two means). $\mathrm{P}<0.05$ was considered to indicate a statistically significant difference.

\section{Results}

MIH treatment attenuates TBI-induced brain edema. The wet-dry weight method was used to evaluate brain edema. As shown in Fig. 1, TBI induced a significant increase in brain edema at 24, 48 and $72 \mathrm{~h}$ compared with the sham group. However, treatment with MIH post-TBI significantly reduced brain edema compared with the TBI group at the corresponding time points.

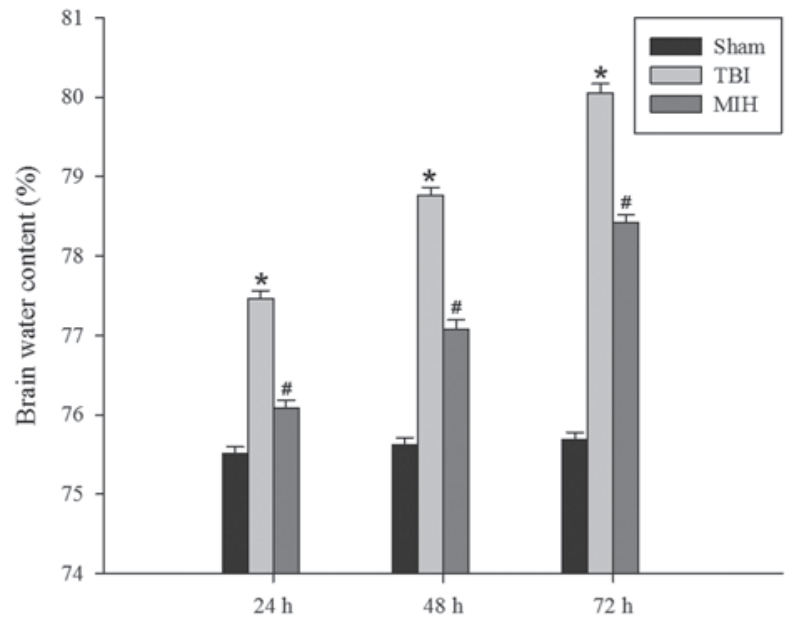

Figure 1. Effect of post-TBI MIH on brain edema. Brain water content was assessed at 24, 48 and $72 \mathrm{~h}$ after TBI and calculated as the percentage of dry and wet weight. Bars represent the mean \pm standard error $(n=6)$. Brain water content significantly increased at 24,48 and $72 \mathrm{~h}$ post TBI. Treatment with MIH for TBI markedly decreased brain water content at 24, 48 and $72 \mathrm{~h}$. ${ }^{*} \mathrm{P}<0.05$, vs. sham group; ${ }^{\#} \mathrm{P}<0.05$, vs. TBI group at the same time point. TBI, traumatic brain injury; MIH, mild induced hypothermia.

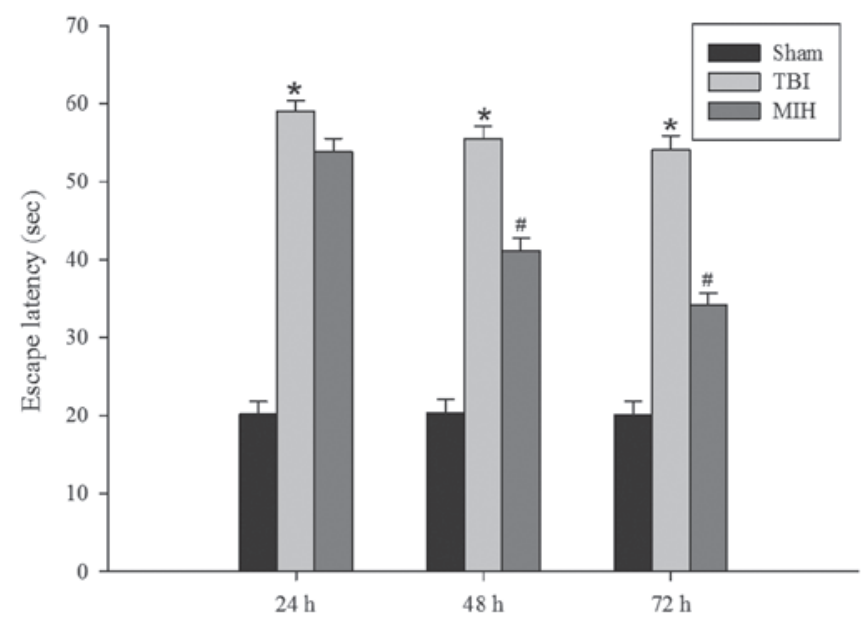

Figure 2. Effect of post-TBI MIH on learning and memory ability. The escape latency performance was determined using a Morris water maze. Bars represent the mean \pm standard error $(n=6)$. The escape latency increased significantly at 24, 48 and $72 \mathrm{~h}$ after TBI. MIH therapy markedly decreased the time to identify the platform at 48 and $72 \mathrm{~h}$. ${ }^{*} \mathrm{P}<0.05$, vs. sham group; ${ }^{\#} \mathrm{P}<0.05$, vs. TBI group at the same time point. TBI, traumatic brain injury; $\mathrm{MIH}$, mild induced hypothermia.

MIH treatment improves learning and memory ability. Following the observation that therapeutic MIH treatment attenuated brain edema post-TBI, the present study next assessed whether MIH was able to improve spatial learning ability using a Morris water maze. As shown in Fig. 2, TBI caused a significant deficit in spatial learning compared with the sham group at 24, 48 and $72 \mathrm{~h}$. However, MIH treatment post-TBI markedly reduced the escape latency compared with the TBI group at 48 and $72 \mathrm{~h}$.

MIH treatment depresses Cx43 protein expression. Immunohistochemical analysis was used to determine the localization of $\mathrm{Cx} 43$ protein. As shown in Fig. 3A, the brown 
A

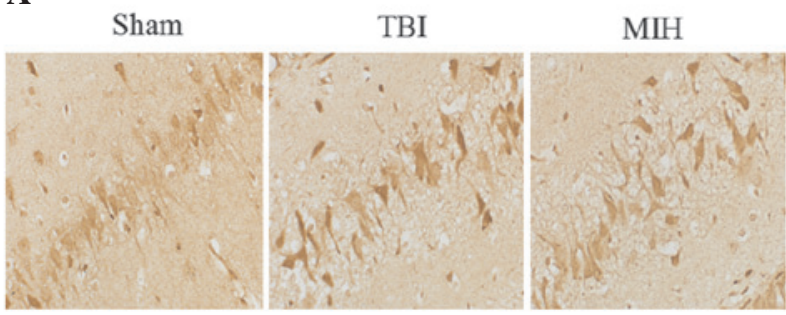

B

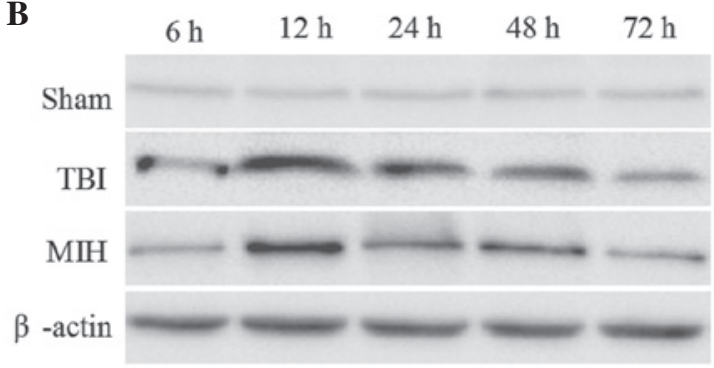

$$
\text { C }
$$

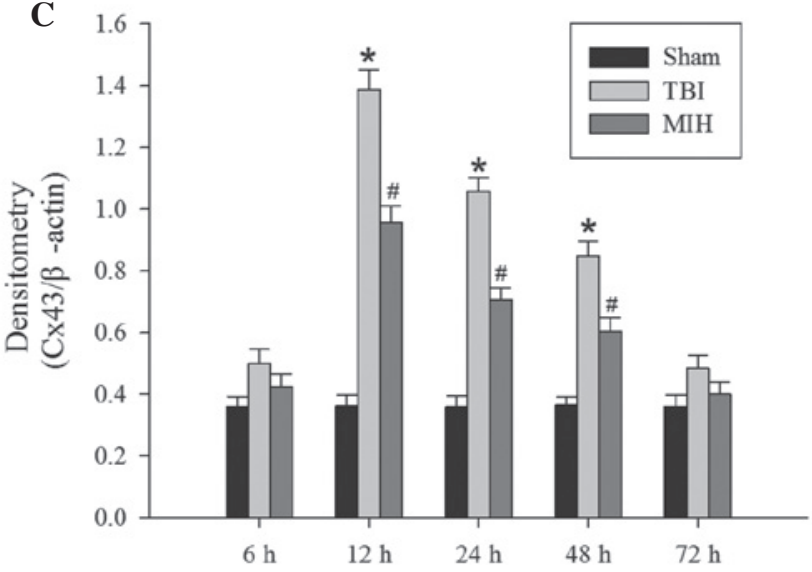

Figure 3. Effect of post-TBI MIH on Cx43 expression. (A) Representative images of immunohistochemical staining of the $\mathrm{Cx} 43$ protein $12 \mathrm{~h}$ post TBI. (B) Western blot analysis of Cx43 bands in the hippocampus and (C) densitometry analysis of bands corresponding to $\beta$-actin. Optical densities of respective protein bands were analyzed with Image J software. The quantitative results of $\mathrm{Cx} 43$ are expressed as the mean of the ratio of densitometries of $\mathrm{Cx} 43$ to $\beta$-actin bands \pm standard error. The expression of $\mathrm{Cx} 43$ significantly increased at 12,24 and $48 \mathrm{~h}$ after TBI. MIH treatment markedly reduced the levels of $\mathrm{Cx} 43$ at 12,24 and $48 \mathrm{~h}$ after TBI. ${ }^{*} \mathrm{P}<0.05$, vs. sham group; ${ }^{*} \mathrm{P}<0.05$, vs. TBI group at the same time point. TBI, traumatic brain injury; $\mathrm{MIH}$, mild induced hypothermia; Cx43, connexin 43.

particles observed in the cells indicated positive results. The majority of brown particles appeared in the astrocytes of the hippocampus, particularly in the membranes of astrocytes and were occasionally expressed in the cytoplasm. Western blot analysis and immunohistochemical analysis revealed the levels of $\mathrm{Cx} 43$ expression in the different groups. As shown in Fig. 3B and C, the protein expression of Cx43 in the hippocampus was present at a consistently low level in the sham group, whereas $\mathrm{Cx} 43$ exhibited a significant increase at different time points in the TBI group. The expression of $\mathrm{Cx} 43$ protein in the hippocampus was increased at $6 \mathrm{~h}$ post TBI, peaked at $12 \mathrm{~h}$ and then gradually decreased, but remained higher than the expression levels in the sham group. Treatment with MIH following TBI caused a significant reduction in the TBI-induced upregulation of $\mathrm{Cx} 43$ expression compared with the TBI group at 12,24 and $48 \mathrm{~h}$.
A

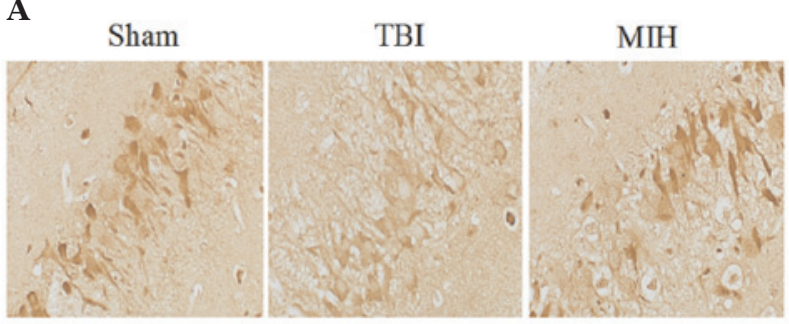

B
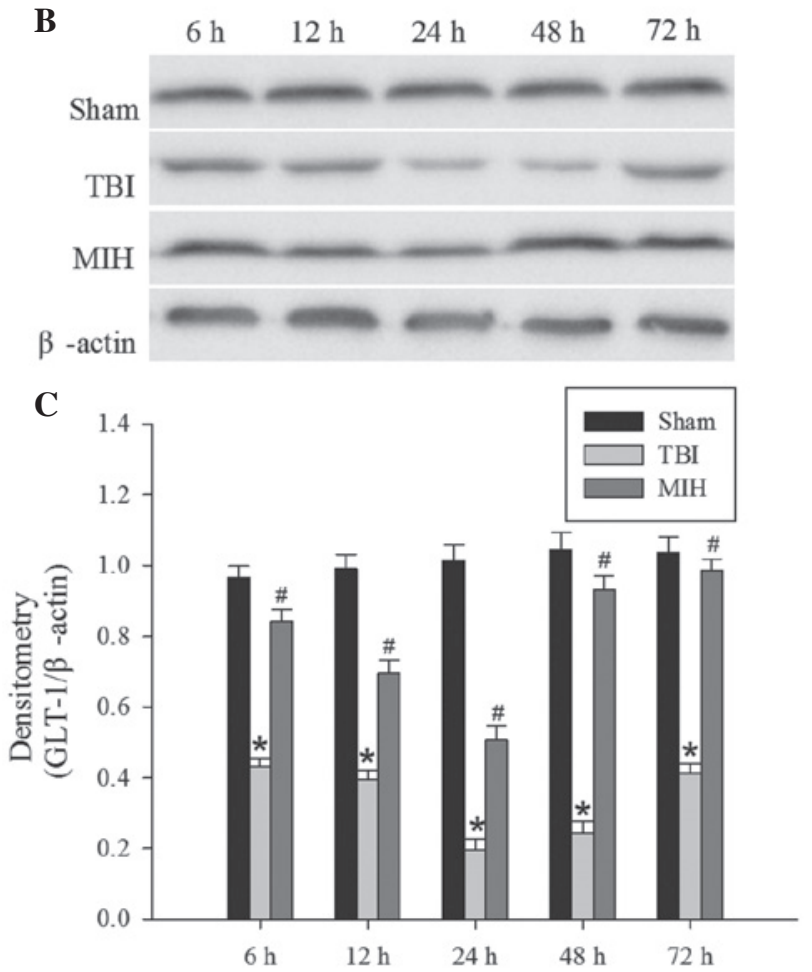

Figure 4. Effect of post-TBI MIH on GLT-1 expression. (A) Representative images of immunohistochemical staining of the GLT-1 protein at $24 \mathrm{~h}$ post TBI. (B) Western blot analysis of GLT-1 bands in the hippocampus and $(C)$ densitometry analysis of bands corresponding to $\beta$-actin. Optical densities of respective protein bands were analyzed with Image $\mathbf{J}$ software. The quantitative results of GLT- 1 are expressed as the mean of the ratio of densitometries of GLT-1 to $\beta$-actin bands \pm standard error. The expression of GLT-1 decreased significantly at 6, 12, 24, 48 and $72 \mathrm{~h}$ after TBI. MIH treatment markedly increased GLT-1 levels following TBI at the corresponding time points. ${ }^{*} \mathrm{P}<0.05$, vs. sham group; ${ }^{\prime} \mathrm{P}<0.05$, vs. TBI group at the same time point. TBI, traumatic brain injury; $\mathrm{MIH}$, mild induced hypothermia; $\mathrm{Cx} 43$, connexin 43; GLT-1, glutamate transporter 1.

MIH treatment upregulates GLT-1 protein expression. The localization of GLT-1 protein was determined by immunohistochemical analysis. GLT-1, a membrane receptor, is predominantly expressed in astrocytes (Fig. 4A). Brown particles were mainly observed in the membranes of astrocytes in the hippocampus and, in part, in the cytoplasm. The levels of GLT-1 were detected by western blot analysis. In the sham group, GLT-1 protein in the hippocampus demonstrated basal expression. As shown in Fig. 4B and C, the expression of GLT-1 was suppressed at $6 \mathrm{~h}$ post TBI, persisted at a low level until $24 \mathrm{~h}$ after TBI and then gradually increased, but remained lower than the GLT-1 expression in the sham group. However, treatment with MIH significantly inhibited the downregulation of GLT- 1 protein compared with the TBI groups at 6,12 , 24,48 and $72 \mathrm{~h}$. 


\section{Discussion}

TBI, an extremely complex neurological condition, leads to acute functional deficit in the brain. However, the molecular events underlying TBI remain to be elucidated. Currently, TBI is a serious health issue with additional social and psychological burdens and the increasing prevalence and high mortality rate of TBI poses a challenge in neurological research. The management of TBI aims to attenuate the amount of secondary brain injury occurring immediately following primary brain insult. Notably, several studies have revealed that MIH treatment following TBI contributes to a reduction in secondary cerebral damage and exerts significant neuroprotective effects (25). In the present study, the neuroprotective action of $\mathrm{MIH}$ was confirmed by reducing brain edema and facilitating the recovery of cognitive function in a rat model of TBI. The results suggested a potential role of $\mathrm{MIH}$ in limiting secondary damage and promoting improved functional outcome following TBI, which is consistent with the results of Marion and Bullock (26) and Dietrich et al (27). Furthermore, immunohistochemical results demonstrated that CX43 was widely located in the membrane of astrocytes and Cx43 was predominantly overexpressed in the hippocampal CA1 following TBI. The results of the present study clearly demonstrated that $\mathrm{Cx} 43$ immunoreactivity was increased in experimental models of brain injury. Cx43-hemichannels remain closed under normal physiological conditions but can open and release glutamate following TBI. Excessive extracellular glutamate following TBI contributes to excitotoxic cell death and is important in the development of secondary injuries (28). However, the levels of glutamate may be effectively controlled by astrocytic glutamate transporters, including GLT-1. The present study revealed that GLT-1 proteins were predominantly localized in astrocytes and were downregulated shortly following TBI. In conclusion, the downregulation of GLT-1 further exacerbated extracellular glutamate accumulation, which resulted in neuronal loss and associated neurological deficits, which have been associated with TBI-induced secondary brain injury in hippocampal tissues (18). Therefore, inhibition of Cx43-hemichannels or upregulation of the expression of GLT-1 can improve neural outcomes, representing novel therapeutic targets for reducing the severity of TBI.

In the present study, the therapeutic potential of $\mathrm{MIH}$ as a neuroprotective strategy for the treatment of TBI was investigated. MIH treatment following TBI was found to be beneficial for neurological recovery. In particular, MIH significantly attenuated brain edema and improved learning and memory ability following TBI in the rat model. However, the underlying mechanisms are yet to be elucidated. The protective effect of hypothermia may involve multiple pathways, including preventing the initiation of apoptotic/necrotic processes, reducing cellular/neuronal damage and inhibiting excitotoxicity (29). An important finding of the present study was that MIH treatment downregulated levels of Cx43 and upregulated the expression of GLT-1 in the hippocampus following TBI. Based on the available evidence, the beneficial effects of MIH in TBI treatment may be explained as follows: $\mathrm{MIH}$ reduced the protein expression of $\mathrm{Cx} 43$ in the astrocytes and decreased the release of extracellular glutamate from
Cx43-hemichannels, or MIH also reversed GLT-1 reduction in the astrocytes and further enhanced the uptake of extracellular glutamate. Taken together, MIH may have the ability to balance glutamate content between intracellular and extracellular environments, therefore having a neuroprotective role following TBI.

MIH treatment exerts a neuroprotective effect on TBI by attenuating brain edema and improving neurological outcomes. These protective effects may be associated with reversing the increase in Cx43 and upregulation of GLT-1 levels following TBI. The present study provided evidence in vivo that MIH has the potential to become a valuable neuroprotective intervention in numerous neurological events.

\section{Acknowledgements}

The present study was supported by a grant from the Science and Technology Development Project of Tangshan City (no. 12140209A-31).

\section{References}

1. Cheng SX, Zhang S, Sun HT and Tu Y: Effects of mild hypothermia treatment on rat hippocampal $\beta$-amyloid expression following traumatic brain injury. Ther Hypothermia Temp Manag 3: 132-139, 2013.

2. Jia F, Mao Q, Liang YM and Jiang JY: Effect of post-traumatic mild hypothermia on hippocampal cell death after traumatic brain injury in rats. J Neurotrauma 26: 243-252, 2009.

3. Fay T: Observations on generalized refrigeration in cases of severe cerebral trauma. Assoc Res Nerv Ment Dis Proc 24: 611-619, 1945.

4. Kramer C, Freeman WD, Larson JS, et al: Therapeutic hypothermia for severe traumatic brain injury: a critically appraised topic. Neurologist 18: 173-177, 2012.

5. Andrews PJ, Sinclair LH, Harris B, et al: Study of therapeutic hypothermia $\left(32\right.$ to $35^{\circ} \mathrm{C}$ ) for intracranial pressure reduction after traumatic brain injury (the Eurotherm3235Trial): outcome of the pilot phase of the trial. Trials 14: 1-8, 2013.

6. Liu L and Yenari MA: Therapeutic hypothermia: neuroprotective mechanisms. Front Biosci 12: 816-825, 2007.

7. Feng JF, Zhang KM, Jiang JY, Gao GY, Fu XA and Liang YM: Effect of therapeutic mild hypothermia on the genomics of the hippocampus after moderate traumatic brain injury in rats. Neurosurgery 67: 730-742, 2010.

8. Laird DW: The gap junction proteome and its relationship to disease. Trends Cell Biol 20: 92-101, 2010.

9. Márquez-Rosado L, Solan JL, Dunn CA, Norris RP and Lampe PD: Connexin43 phosphorylation in brain, cardiac, endothelial and epithelial tissues. Biochi Biophys Acta 1818: 1985-1992, 2012.

10. Mroue R, El-Sabban M and Talhouk R: Connexins and the gap in context. Integ Biol 3: 255-266, 2011.

11. Ongstad EL, O'Quinn MP, Ghatnekar GS, Yost MJ and Gourdie G: A connexin43 mimetic peptide promotes regenerative healing and improves mechanical properties in skin and heart. Adv Wound Care (New Rochelle) 2: 55-62, 2013.

12. Yu M, Zhang C, Li L, Dong S, Zhang N and Tong X: Cx43 reverses the resistance of A549 lung adenocarcinoma cells to cisplatin by inhibiting EMT. Oncol Rep 31: 2751-2758, 2014.

13. Sun LQ, Gao JL, Cui CM, et al: Astrocytic p-connexin43 regulates neuronal autophagy in the hippocampus following traumatic brain injury in rats. Mol Med Rep 9: 77-82, 2014.

14. Ohsumi A, Nawashiro H, Otani N, Ooigawa H, Toyooka T and Shima K: Temporal and spatial profile of phosphorylated connexin43 after traumatic brain injury in rats. J Neurotrauma 27: 1255-1263, 2010.

15. Rami A, Volkmann T and Winckler J: Effective reduction of neuronal death by inhibiting gap junctional intercellular communication in a rodent model of global transient cerebral ischemia. Exp Neurol 170: 297-304, 2001.

16. Jiang S, Wang YQ, Xu CF, Li YN, Guo R and Li L: Involvement of connexin43 in the infrasonic noise-induced glutamate release by cultured astrocytes. Neurochem Res 39: 833-842, 2014. 
17. Obrenovitch TP, Urenjak J: Is high extracellular glutamate the key to excitotoxicity in traumatic brain injury? J Neurotrauma 14: 677-698, 1997.

18. Yi JH, Hazell AS. Excitotoxic mechanisms and the role of astrocytic glutamate transporters in traumatic brain injury. Neurochem Int 48: 394-403, 2006.

19. Wang X, Ma A, Zhu W, et al: The role of connexin 43 and hemichannels correlated with the astrocytic death following ischemia/reperfusion insult. Cell Mol Neurobiol 33: 401-410, 2013.

20. Goodrich GS, Kabakov AY, Hameed MQ, Dhamne SC, Rosenberg PA and Rotenberg A: Ceftriaxone treatment after traumatic brain injury restores expression of the glutamate transporter, GLT-1, reduces regional gliosis and reduces post-traumatic seizures in the rat. J Neurotrauma 30: 1434-1441, 2013.

21. Marmarou A, Foda MA, van den Brink W, Campbell J, Kita H and Demetriadou K: A new model of diffuse brain injury in rats: Part I: Pathophysiology and biomechanics. J Neurosurg 80: 291-300, 1994.

22. Truettner JS, Alonso OF, Bramlett HM and Dietrich WD: Therapeutic hypothermia alters microRNA responses to traumatic brain injury in rats. J Cereb Blood Flow Metab 31: 1897-1907, 2011.
23. Tang J, Liu J, Zhou C, et al: Mmp-9 deficiency enhances collagenase-induced intracerebral hemorrhage and brain injury in mutant mice. J Cereb Blood Flow Metab 24: 1133-1145, 2004.

24. Hui-guo L, Kui L, Yan-ning Z and Yong-jian X: Apocynin attenuate spatial learning deficits and oxidative responses to intermittent hypoxia. Sleep Med 11: 205-212, 2010.

25. Tokutomi T, Morimoto K, Miyagi T, Yamaguchi S, Ishikawa K and Shigemori M: Optimal temperature for the management of severe traumatic brain injury: effect of hypothermia on intracranial pressure, systemic and intracranial hemodynamics, and metabolism. Neurosurgery 52: 102-112, 2003.

26. Marion D and Bullock MR: Current and future role of therapeutic hypothermia. J Neurotrauma 26: 455-467, 2009.

27. Dietrich WD, Atkins CM and Bramlett HM: Protection in animal models of brain and spinal cord injury with mild to moderate hypothermia. J Neurotrauma 26: 301-312, 2009.

28. Obrenovitch TP and Urenjak J: Is high extracellular glutamate the key to excitotoxicity in traumatic brain injury? J Neurotrauma 14: 677-698, 1997.

29. Broessner G, Fischer M, Schubert G, Metzler B and Schmutzhard E: Update on therapeutic temperature management. Critical Care 16: A1, 2012. 